\title{
Three Dimensional Assembly in Directed Self-assembly of Block Copolymers
}

\author{
Tamar Segal-Peretz ${ }^{1,2}$, Chun Zhou ${ }^{1}$, Jiaxing Ren ${ }^{1}$, Takahiro Dazai ${ }^{3}$, Leonidas E. Ocola ${ }^{4}$, \\ Ralu N. S. Divan ${ }^{4}$, and Paul F. Nealey ${ }^{1,2}$ \\ ${ }^{1}$ Institute for Molecular Engineering, University of Chicago, 5747 South Ellis Ave, Chicago, \\ 60637, United States \\ ${ }^{2}$ Materials Science Division, Argonne National Laboratory, 9700 S. Cass Avenue, Argonne, \\ Illinois 60439, United States \\ ${ }^{3}$ Tokyo Ohka Kogyo Co., Ltd.1590 Tabata, Samukawa-machi, Koza-gun, Kanagawa-ken 253-0114, \\ Japan \\ ${ }^{4}$ Center for Nanoscale Materials, Argonne National Laboratory, 9700 S. Cass Avenue, Argonne, \\ Illinois 60439, United States
}

\begin{abstract}
The three-dimensional assembly of poly (styrene- $b$-methyl methacrylate) (PS- $b$ PMMA) in chemoepitaxy and graphoepitaxy directed self-assembly (DSA) was investigated using scanning transmission electron microscopy (STEM) tomography. The tomographic characterization revealed hidden morphologies and defects at the BCP- chemical pattern interface in lamellar DSA, and probed the formation of cylinders at the bottom of cylindrical DSA for contact hole shrink. Future work will include control over 3D assembly in sub-10 nm processes.

Keywords: Directed self-assembly, block copolymer, lithography, contact hole, transmission electron microscopy, metrology
\end{abstract}

\section{Introduction}

Directed self-assembly (DSA) of block copolymer (BCP) films has gained extensive interest in the last few years due to its ability to extend the lithographic capabilities beyond $193 \mathrm{~nm}$ immersion lithography [1,2]. A variety of directing strategies, such as chemoepitaxy $[3,4]$, graphoepitaxy $[5,6]$, and pre-patterns that combine both chemo and graphoepitaxy [7], have been demonstrated. While BCP thin films are generally addressed as 2D patterning layers, BCP are inherently three dimensional (3D) materials, and even in extremely thin film, where the film thickness is smaller than the BCP periodicity $\left(\mathrm{L}_{0}\right), 3 \mathrm{D}$ structures can be found [8]. Indeed recent investigations using $\mathrm{X}$-ray scattering $[9,10]$, transmission electron microscopy (TEM) $[8,11]$, and molecular simulations $[12,13]$ have shed light on the three-dimensional structures that form in DSA films. These structures include the through-film domain morphology, the fluctuations (roughness) of the domains, and the $3 \mathrm{D}$ structure of defects. Understanding and controlling the BCP assembly at 3D becomes even more important as nanomanufacturing is moving towards sub-10 $\mathrm{nm}$ features.

Current characterization of DSA film heavily relies upon 2D top-down or cross sectional imaging. However, 2D imaging is not sufficient to fully describe the DSA morphology and 3D characterization is needed to better understand the assembly process and to probe hidden morphologies under the surface. In particular, controlling $\mathrm{BCP}$ assembly at the $\mathrm{BCP}$-guiding pattern interface is crucial for obtaining DSA for line-space patterns with high degree of registration and perfection, as well as for contact hole shrink 
(a)
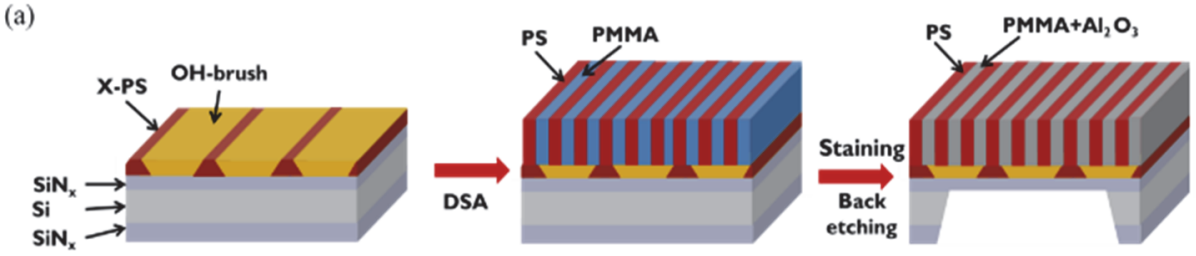

(b)
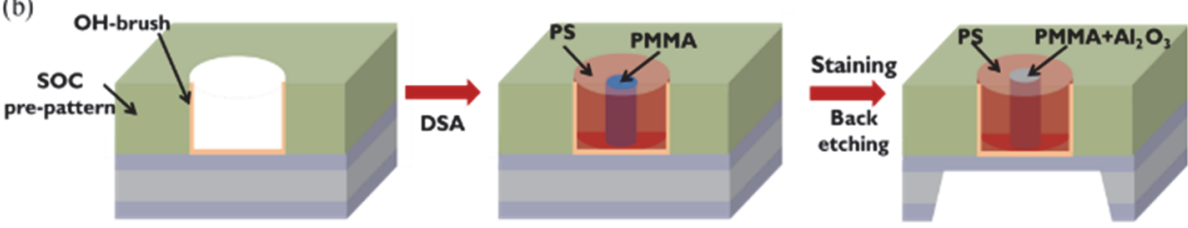

(c)
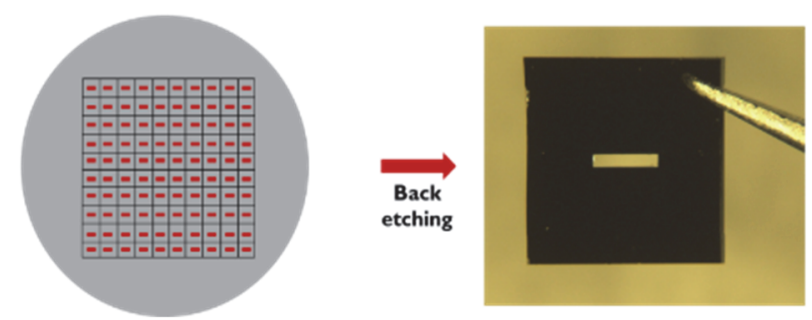

Fig. 1. Schematic illustration of sample preparation for 3D characterization: (a) DSA of lamellae-forming PS- $b$ PMMA on chemical pattern with $3 \mathrm{X}$ density multiplication, and (b) DSA of cylinder-forming PS- $b$-PMMA on SOC pre-pattern for contact hole shrink. Following the DSA, PMMA was selectively stained using $\mathrm{Al}_{2} \mathrm{O}_{3} \mathrm{SIS}_{\text {to enhance }}$ the imaging contrast. (c) The silicon wafer was back etched to fabricate $\mathrm{Si} / \mathrm{SiN}_{\mathrm{x}}$ windows for TEM characterization.

process where the assembly at the bottom interface determines the contact properties.

In this research, we utilize scanning TEM (STEM) tomography to investigate the three dimensional assembly in poly (styrene-blockmethyl methacrylate) (PS- $b$-PMMA) DSA films in two processes: DSA of lamellaeforming PS- $b$-PMMA on chemical patterns (Figure 1a) for line-space patterns, and DSA of cylinder-forming PS- $b$-PMMA on graphoepitaxy pre-patterns for contact hole shrink (Figure 1b). We were able to probe curved lamellae and defects at the BCPchemical pattern interface, and PS residual layer at the cylindrical DSA.

\section{Methods}

In order to enable 3D characterization with STEM tomography, samples were prepared on 4" $\mathrm{SiN}_{\mathrm{x}} / \mathrm{Si} / \mathrm{SiN}_{\mathrm{x}}$ wafers $(30 \mathrm{~nm} / 200 \mathrm{~nm} / 30 \mathrm{~nm})$ that could be back-etched to create $100,3 \mathrm{~mm}$ x $3 \mathrm{~mm}$, individual samples with $0.5 \mathrm{~mm} \times 200$ $\mu \mathrm{m} \mathrm{SiN}_{\mathrm{x}}$ windows for TEM imaging (Figure 1c). Optical lithography was performed on the back side of the wafer to define the individual sample dimensions and the openings in the back-side $\mathrm{SiN}_{\mathrm{x}}$ [11]. For lamellar DSA, chemical patterns were fabricated according to the Liu-Nealey (LiNe) flow:4 $84 \mathrm{~nm}$ pitch, cross-linkable poly(styrene) (X-PS) lines were patterned using e-beam lithography, and the pattern was backfilled with hydroxylterminated $\mathrm{P}(\mathrm{S}-r$-MMA)-OH brush. Following the chemical pattern fabrication, lamellaforming PS- $b$-PMMA (AZEMBLYTM PME312, lamellae spacing $\mathrm{L}_{0}=28 \mathrm{~nm}$ ) was spin coated on the chemical template and annealed at $250{ }^{\circ} \mathrm{C}$ for $5 \mathrm{~min}$. For contact hole shrink DSA, $100 \mathrm{~nm}$ thick spin-on-carbon (SOC) prepatterns were fabricated using e-beam lithography. The pre-pattern holes had diameter of $60 \mathrm{~nm}$ and were patterned in 110 $\mathrm{nm}$ pitch. Cylinder-forming PS- $b$-PMMA (provided by TOK, BCP spacing $\mathrm{L}_{0}=33 \mathrm{~nm}$ ) was spin coated on the SOC pre-pattern and annealed at $230{ }^{\circ} \mathrm{C}$ for $10 \mathrm{~min}$.

To fabricate the TEM samples, the 4" wafers were back-etched in $\mathrm{KOH}$ solution for $\sim 3$ hours while the front side was sealed and protected by a wet etch holder. Following the back-etch process, the individual samples were stained using selective growth of $\mathrm{Al}_{2} \mathrm{O}_{3}$ in the PMMA domain using sequential infiltration synthesis (SIS) process $[14,15]$. SIS process, which enables growth of metal oxides inside polymer and BCP films with high selectivity to BCP polar domains [16], can be used to enhance the etch contrast between the BCP 
domains and improve pattern transfer [17,18]. It was recently shown that SIS is also an excellent staining process for BCPs since it results in high imaging contrast and it is highly stable under the electron beam $[14,15,19]$. The $\mathrm{BCP}$ films were stained with $\mathrm{Al}_{2} \mathrm{O}_{3}$ SIS using $\mathrm{Al}\left(\mathrm{CH}_{3}\right)_{3}$ and $\mathrm{H}_{2} \mathrm{O}$ precursors at $95{ }^{\circ} \mathrm{C}$.

STEM tomography was performed using a field-emission gun TEM operated at $200 \mathrm{kV}$. Tilt series were acquired by tilting the samples between $-70^{\circ}$ to $+70^{\circ}$ with $2^{\circ}$ to $3^{\circ}$ intervals, resulting in 51 STEM images per tilt series. The tilt series were then aligned and reconstructed using simultaneous iterative reconstruction technique (SIRT) algorithm to resolve the $3 \mathrm{D}$ structure. Thresholding and visualization were performed using ImageJ and FEI software (AvizuTM).

\section{Results and Discussion}

3.1. Lamellar DSA for line-space patterns AZEMBLYTM PME312 has been shown to have a large process window on chemical pattern with $3 \mathrm{X}$ density multiplication, assembling with high degree of perfection (as evaluated by SEM imaging) at X-PS guiding stripes widths (W) between $0.6 \mathrm{~L}_{0}$ and $0.9 \mathrm{~L}_{0}$ $\mathrm{m}$ [20]. To examine the 3D assembly of AZEMBLYTM PME312 in and around the process window, chemical patterns with $\mathrm{W}$ values ranging between $0.5 \mathrm{~L}_{0}$ to $1.5 \mathrm{~L}_{0}$ were fabricated. Figure 2 show tomographic characterization of AZEMBLYTM PME312 assembly on chemical pattern with $\mathrm{W}=0.8 \mathrm{~L}_{0}$. Visualization of the reconstructed volume (Figure 2a) highlights the rich 3D morphological data in a DSA film, showing both the through-film structure and the fluctuation of this structure across the $900 \mu \mathrm{m}$ x $900 \mu \mathrm{m} \quad$ x $30 \mu \mathrm{nm} \quad(\mathrm{x}, \mathrm{y}, \mathrm{z})$ reconstruction volume. To study the through-film morphology in details, the tomographic reconstruction volume was sliced to $1800,0.5 \mathrm{~nm}$ thick, cross sections (xz), which were averaged to obtained an averaged cross section (Figure $2 b$ ). Bright domains in the STEM images correspond to PMMA domains stained with $\mathrm{Al}_{2} \mathrm{O}_{3}$, while dark domains correspond to PS domains. The average cross section shows that while PS-bPMMA assembles in perpendicular and aligned assembly throughout most of the film thickness, curved lamellae are seen close to the interface with the chemical pattern. As can be seen from the different contrast between the XPS and the brush domain (X-PS is illustrated in red trapezoid), the curved lamellae are adjacent to the guiding stripe walls. The curvature is the result of the X-PS geometry and the favorable interaction of the PMMA with the guiding stripes walls. Curved lamellae can reduce the quality of the pattern transfer features and lead to line placement errors.

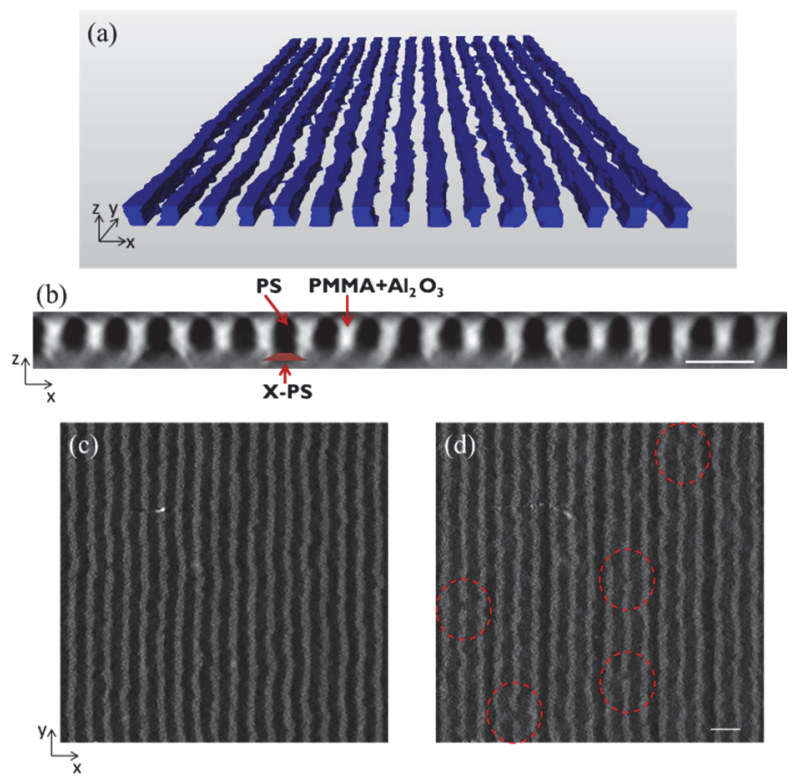

Fig. 2. STEM tomography of lamellar PS-b-PMMA DSA on chemical pattern with $3 \mathrm{X}$ density multiplication: (a) visualization of the reconstructed volume. For clarity, PMMA is colored blue, while PS is transparent. (b) average cross section obtained from the reconstruction volume. (c,d) two slices, parallel to the substrate, from the reconstructed volume showing the assembly at the top of the film (c) and at the BCPchemical pattern interface (d); dashed red circles show line-breakage defects. Scale bars are $50 \mathrm{~nm}$.

Parallel to the substrate (xy) slices from the reconstruction volume show the assembly at the BCP-free surface interface and the BCPchemical pattern interface, Figures $2 \mathrm{c}$ and $2 \mathrm{~d}$, respectively. The $\mathrm{xy}$ slices show that while defect-free assembly is seen at the top of the film (Figure 2c), several line-breakage defects are seen at the bottom of the film (Figure $2 \mathrm{~d}$, defects are marked with dashed red circles). Inspection of the defects' location relatively to the chemical pattern features revealed that all the line-breakage in the field of view are over the brush domain, indicating a preference of this defect to the background region. PMMA line-breakage defects can also been seen as PS micro-bridge defects. Their location over the 
brush domain has recently been associated with the preferential wetting of PMMA by the X-PS side walls that suppresses micro-bridge formation over the guide stripes [21], in good agreement with the defects seen in this research.

\subsection{Cylindrical DSA for contact hole shrink}

The assembly of PS- $b$-PMMA in contact hole pre-patterns was investigated using STEM tomography. Figure 3 shows visualization of 9 contact holes with cylinderforming PS- $b$-PMMA assembly (Figure 3a) and $0.5 \mathrm{~nm}$ thick, cross section of the reconstructed volume (Figure $3 \mathrm{~b}$ ). In each hole, the inner bright domain corresponds to $\mathrm{Al}_{2} \mathrm{O}_{3}$ stained PMMA cylinders while the outer bright domain corresponds to the $\mathrm{Al}_{2} \mathrm{O}_{3}$-stained PS$\mathrm{OH}$ brush which was used to control the surface chemistry of the SOC holes, highlighting the SOC hole outline. PS and the SOC had similar contrast in the STEM images, seeing here as dark domains. To assist in the visualization, the PMMA cylinders are colored in blue, the PS-OH brush is colored in red, while the PS and the SOC are transparent. The tomographic data clearly shows that while the PMMA assembles in perpendicular cylinder at the center of the SOC hole, it does not reach the bottom of the SOC pre-pattern and a $\sim 10$ $\mathrm{nm}$ thick PS layer exists at the bottom of the hole. This layer originates from the PS-wetting

(a)

(b)
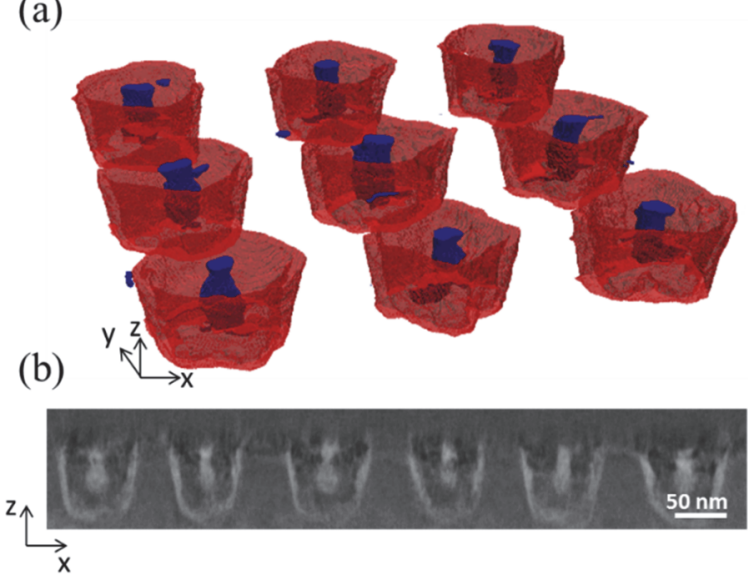

Fig. 3. STEM tomography of hole shrink DSA: (a) visualization of the reconstructed volume. For clarity, $\mathrm{Al}_{2} \mathrm{O}_{3}$-stained PMMA is colored in blue, $\mathrm{Al}_{2} \mathrm{O}_{3}$-stained brush is colored in red, while PS and the SOC pre-pattern are transparent. (b) Digitally sliced cross section obtained from the reconstructed volume. brush that was coated on both the walls and the bottom of the pre-pattern hole.

\section{Conclusion}

The 3D morphology of lamellae and cylindrical DSA films was characterized using STEM tomography. Both hidden defects and the morphology at the $\mathrm{BCP}$-guiding pattern interface were probed. This research demonstrates the importance of understating the 3D assembly in DSA films and how 3D characterization enables to investigate the impact of the polymer chemistry, the geometry and chemistry of the guiding pattern, and the surface properties on $\mathrm{BCP}$ assembly.

\section{Acknowledgements}

This work was supported by the U.S. Department of Energy, Office of Science, Office of Basic Energy Sciences, at the Materials Science Division and at the Center for Nanoscale Materials, in Argonne National Laboratory under Contract No. DE-AC0206CH11357. We gratefully acknowledge the University of Chicago-Searle Cleanroom for nanofabrication services.

\section{References}

1. C. M. Bates, M. J. Maher, D. W. Janes, C. J. Ellison, C. G. Willson, Macromolecules, 47 (2014) 2.

2. M. P. Stoykovich, P. F. Nealey, Mater. Today, 9 (2006) 20.

3. S. O. Kim, H. H. Solak, M. P. Stoykovich, N. J. Ferrier, J. J. de Pablo, P. F. Nealey, Nature, 424 (2003) 411.

4. C.-C. Liu, E. Han, M. S. Onses, C. J. Thode, S. Ji, P. Gopalan, P. F. Nealey, Macromolecules, 44 (2011) 1876.

5. I. Bita, J. K. W. Yang, Y. S. Jung, C. A. Ross, E. L. Thomas, K. K. Berggren, Science, 321 (2008) 939.

6. R. Ruiz, R. L. Sandstrom, C. T. Black, $A d v$. Mater., 19 (2007) 587.

7. J. D. Cushen, L. Wan, G. Blachut, M. J. Maher, T. R. Albrecht, C. J. Ellison, C. G. Willson, R. Ruiz, ACS Appl. Mater. Interfaces, 7 (2015) 3323.

8. K. W. Gotrik, T. Lam, A. F. Hannon, W. Bai, Y. Ding, J. Winterstein, A. Alexander-Katz, J. A. Liddle, C. A. Ross, Adv. Funct. Mater, 24 (2014) 7689. 
9. G. E. Stein, J. A. Liddle, A. L. Aquila, E. M. Gullikson, Macromolecules, 43 (2010) 433.

10. D. F. Sunday, M. R. Hammond, C. Wang, W.-1. Wu, D. M. Delongchamp, M. Tjio, J. Y. Cheng, J. W. Pitera, R. J. Kline, ACS Nano, 8 (2014) 8426.

11. T. Segal-Peretz, J. Winterstein, J. Ren, M. Biswas, J. A. Liddle, J. W. Elam, L. E. Ocola, R. N. S. Divan, N. Zaluzec, P. F. Nealey, Proc. SPIE, 9424 (2015) 94240U.

12. S.-M. Hur, V. Thapar, A. Ramírez-Hernández, G. Khaira, T. Segal-Peretz, P. A. Rincon-Delgadillo, W. Li, M. Müller, P. F. Nealey, J. J. de Pablo, Proc. Natl. Acad. Sci. U.S.A., 112 (2015) 14144.

13. W. Li, M. Müller, Prog. Polym. Sci., 54-55 (2016) 47-75.

14. T. Segal-Peretz, J. Winterstein, M. Biswas, J. A. Liddle, J. W. Elam, N. J. Zaluzec, P. F. Nealey, Microsc. Microanal., 21 (2015) (SupplementS3), 611.

15. T. Segal-Peretz, J. Winterstein, M. Doxastakis, A. Ramírez-Hernández, M. Biswas, J. Ren, H. S. Suh, S. B. Darling, J. A. Liddle, J. W. Elam, J. J. de Pablo, N. J. Zaluzec, P. F. Nealey, ACS Nano, 9 (2015) 5333.

16. Q. Peng, Y.-C. Tseng, S. B. Darling, J. W. Elam, Adv. Mater., 22 (2010) 5129.

17. R. Ruiz, L. Wan, J. Lille, K. C. Patel, E. Dobisz, D. E. Johnston, K. Kisslinger, C. T. Black, J. Vac. Sci. Technol. B, 30 (2012) 06F202.

18. A. Singh, W. Knaepen, S. Sayan, Z. el Otell, B. T. Chan, J. W. Maes, R. Gronheid, Proc. SPIE, 9425 (2015) $94250 \mathrm{~N}$.

19. J. W. Elam, M. Biswas, S. Darling, A. YanguasGil, J. D. Emery, A. B. F. Martinson, P. F. Nealey, T. Segal-Peretz, Q. Peng, J. Winterstein, J. A. Liddle, Y.-C. Tseng, ECS Transactions, 69 (2015) 147.

20. P. A. R. Delgadillo, R. Gronheid, C. J. Thode, H. $\mathrm{Wu}$, Y. Cao, G. Lin, M. Somervell, K. Nafus, P. F. Nealey, J. Photopolym. Sci. Technol., 25 (2012) 77.

21. L. D. Williamson, R. N. Seidel, X. Chen, H. S. Suh, P. Rincon Delgadillo, R. Gronheid, P. F. Nealey, ACS Appl. Mater. Interfaces, 8 (2016) 2704. 